\title{
Residing near allergenic trees can increase risk of allergies later in life: LISA Leipzig study
}

Iana Markevych ${ }^{1}$, Romina Ludwig ${ }^{2}$, Clemens Baumbach ${ }^{3}$, Marie Standl ${ }^{4}$, Joachim

Heinrich $^{5}$, Gunda Herberth ${ }^{6}$, Kees de Hoogh ${ }^{7}$, Karin Pritsch ${ }^{8}$, and Fabian Weikl ${ }^{8}$

${ }^{1}$ Jagiellonian University

${ }^{2}$ Institute and Clinic for Occupational, Social and Environmental Medicine, University

Hospital, LMU Munich

${ }^{3}$ ENIANO GmbH

${ }^{4}$ Helmholtz Zentrum München

${ }^{5}$ Ludwig-Maximilians-Universitat Munchen Medizinische Fakultat

${ }^{6}$ UFZ-Centre for Environmental Research

${ }^{7}$ Swiss Tropical and Public Health Institute

${ }^{8}$ Allergens in Ecosystems, Institute of Biochemical Plant Pathology, Helmholtz Zentrum

München - German Research Center for Environmental Health

June 4, 2020

\begin{abstract}
Background: We investigated whether residing in places with higher greenness, more trees and more allergenic trees early in life increases the risk of allergic outcomes, and whether these associations differ depending on the concentration of air pollutants. Methods: The analytic sample included 631 children from the German birth cohort LISA Leipzig. Asthma and allergic rhinitis, sensitization to aeroallergens and food allergens, as well as confounders, were collected prospectively up to 15 years. Greenness was assessed by Normalized Difference Vegetation Index (NDVI). A tree registry was used to derive information on trees, which were classified into allergenic and non-allergenic. Annual average concentrations of nitrogen dioxide (NO2) and ozone were also used. Geographic exposures were assigned to home addresses at birth. Longitudinal associations were analysed using generalized estimating equations. Results: Medium and high numbers (tertiles) of trees and allergenic trees in a $500 \mathrm{~m}$ buffer around birth addresses were associated with increased odds of allergic rhinitis up to 15 years regardless of NDVI. These exposures were also related to higher odds of sensitization to aeroallergens. Associations with asthma and sensitization to food allergens were less consistent. Effect estimates for allergic rhinitis were stronger in the high tertile of NO2 compared to the low tertile, while an opposite tendency was observed for ozone. Conclusion: We observed that early life residence in places with many trees, and allergenic trees specifically, may increase the prevalence of allergic rhinitis later in life. This association and its modification by air pollution should be pursued in further studies.
\end{abstract}

\section{Abbreviations}

\begin{tabular}{ll}
\hline CI & Confidence interval \\
\hline ELAPSE & Effects of Low-Level Air Pollution: A Study in Europe \\
IgE & Immunoglobulin E \\
GAM & Generalized additive model \\
GEE & Generalized estimating equation \\
LISA & Influence of Life-Style Factors on the Development of the Immune System and Allergies in East and West Germ
\end{tabular}




\begin{tabular}{ll}
\hline CI & Confidence interval \\
\hline LUR & Land use regression \\
$\mathrm{NDVI}$ & Normalized Difference Vegetation Index \\
$\mathrm{NO}_{2}$ & Nitrogen dioxide \\
$\mathrm{OR}$ & Odds ratio \\
\hline
\end{tabular}

\section{Introduction}

Greenspace is related to various beneficial health outcomes, including better mental health and well-being ${ }^{1}$, reduced risk of all-cause mortality ${ }^{2}$ and type 2 diabetes ${ }^{3}$, improved pregnancy outcomes and decreased blood pressure ${ }^{4}$, among others. These benefits are hypothesized to be due to the restorative potential of greenspace, reduced concomitant exposure to harmful environmental influences, as well as because greenspace provides settings for physical activity and social contacts which in turn improve physical and mental health. ${ }^{5}$

Mechanistic links between greenspace, asthma and allergic outcomes seem to be more complicated to resolve than those for other health indicators. On the one hand, greenspace was hypothesized to reduce the risk of allergic diseases by similar mechanisms as does living on a farm, ${ }^{6}$ namely via increased contact with microbes and consequent modulating of immune responses and allergic inflammation. ${ }^{7}$ On the other hand, greenspace is a source of allergenic pollen, short-term exposure to which can exacerbate asthma and allergic symptoms as studies based on pollen counts have demonstrated. ${ }^{8}$

It is not surprising then that greenspace research into allergic health outcomes stagnates. The published studies have typically reported associations, but those were in different directions depending on the study area. ${ }^{9,10}$ One example for such diverging directions is our own study where higher greenness (i.e., vegetation degree) was associated with increased risk of allergic rhinoconjunctivitis and aeroallergen sensitization in the Munich study area, but with reduced risk of these outcomes in the Wesel area of the same multicentre birth cohort. ${ }^{11}$ We failed to explain such inconsistency but managed to replicate it in five additional cohorts across three continents. ${ }^{12}$

Besides the complicated and potentially conflicting underlying mechanisms, the lack of consistency in greenspace-allergy studies was also blamed on metrics of greenspace that are too crude to differentiate between different types of vegetation. ${ }^{12}$ Most of the existing research used either land use or land coverderived distance to or amount of different types of green spaces, or satellite-derived vegetation indices capturing greenness. None of these metrics can distinguish trees from herbaceous vegetation, let alone allergenic from non-allergenic plant species.

Keeping all this in mind, we decided to explore whether residing in places with overall higher greenness, more trees and specifically more allergenic trees early in life can contribute to the onset of allergic diseases, or whether such exposures act as protective factors. To our knowledge, no study so far looked at the longterm exposure to allergenic trees and development of allergic diseases - partially because detailed vegetation geodata are very scarce and partially because expert knowledge is needed to classify species into allergenic and non-allergenic. This study was possible thanks to the collaboration between environmental epidemiologists and plant biologists.

Since elevated air pollution levels are observed to increase the allergenicity of pollen, ${ }^{13}$ as a secondary objective, we investigated whether the associations between greenness, trees and allergenic trees and allergic diseases differ depending on the concentration of several air pollutants. In addition, vegetation and air pollution are inversely spatially correlated and, depending on the spatial arrangement, composition and temperature, trees can remove, trap, or even emit air pollution. ${ }^{14}$

\section{Methods}

\section{Study design and population}


"Influence of $\mathbf{L}$ ife-Style Factors on the Development of theI mmune $\mathbf{S}$ ystem and $\mathbf{A}$ llergies in East and West Germany" (LISA) is an ongoing multicentre population-based German birth cohort. 15,16 Briefly, healthy full-term neonates with a normal birth weight were recruited at selected maternity hospitals in Munich, Leipzig, Wesel and Bad Honnef between 1997 and 1999. Parent-completed questionnaires were administered at birth and at ages $0.5,1,1.5,2,4,6,10$ and 15. Blood samples were drawn from a subset of children at the ages of 2, 6, 10 and 15 years. For the purpose of this study, we utilized the data from 631 children from Leipzig (for details, see the flowchart, Figure E1). The LISA Leipzig cohort was approved by the local ethics committee at the University of Leipzig (560/1998; 206/2003; 345/2007) and the State Medical Council of Saxony (EK-BR-02/13-1), and informed consent was obtained from all families.

\section{Allergic outcomes}

Data on parent-reported doctor diagnosis of asthma and allergic rhinitis (AR) were collected at ages $0.5,1$, 1.5, 2, 4, 6, 10 and 15. If the time between follow-ups was longer than one year (e.g., 10 to 15 years), the presence of the diagnosis was asked separately for each year since the previous follow-up (e.g., 10, 11, 12, 13, 14 and 15). In line with our previous analyses, ${ }^{17}$ only information collected from age 3 onwards was used due to difficulty of accurate diagnosis of these outcomes at younger ages. Asthma at ages $\mathrm{Y}=3,4, \ldots 15$ was defined as a positive response to "Was your child diagnosed with asthma by a doctor at the age of $\mathrm{Y}$ years?" AR at ages $\mathrm{Y}=3,4, \ldots 15$ was defined as a positive response to "Was your child diagnosed with hay fever/allergic rhinitis by a doctor at the age of Y years?"

Specific immunoglobulin E (IgE) against common allergens was assessed at ages 2, 6, 10 and 15 using the standardized CAP-RAST FEIA method (ThermoFischer, Freiburg, Germany). At 2 years, allergic sensitization was tested to tree and grass pollen (RX1), mold, cat and house dust mites. At 6 , 10 and 15 years, allergic sensitization was tested by using inhalant mix SX1, which consists of house dust mite, cat, dog, mold, birch, rye, mugwort and timothy grass allergens. Allergic sensitization to aeroallergens was defined as $\operatorname{IgE}>0.35 \mathrm{kU} / \mathrm{L}$ for at least one of the tested inhalant allergens at 2 years and for the SX1 mix at the other timepoints. Allergic sensitization to food allergens was defined as $\operatorname{IgE}>0.35 \mathrm{kU} / \mathrm{L}$ for allergens in the FX5 screening test (milk, peanut, eggs, soya, cod and wheat flour) at all timepoints.

\section{Greenness, trees and allergenic trees}

The Normalized Difference Vegetation Index (NDVI) ${ }^{18}$ was used as an indicator of greenness (i.e. vegetation degree). NDVI is constructed from the ratio of reflected light to absorbed light in two vegetation-informative bands of the electromagnetic spectrum: the red band (RED) and the near infrared band (NIR). NDVI is calculated as NDVI $=(\mathrm{NIR}-\mathrm{RED}) /(\mathrm{NIR}+\mathrm{RED})$. Thus, NDVI ranges from -1 (water) through 0 (barren areas) to +1 (areas completely covered by vegetation).

For our NDVI calculations, we used a single cloud-free Landsat 5 TM satellite image at a resolution of 30 by $30 \mathrm{~m}$ taken on June 6, 1997 (https://earthexplorer.usgs.gov/) which is about the time when recruitment for the study started. Summer images enable maximum spatial contrasts of greenness. 19

Tree registry data of the city of Leipzig were utilized to derive information on trees. Briefly, data are available for street trees and trees in green spaces and include tree geocoordinates, tree species in binomial and German nomenclature and year of planting. Only trees planted before 1998 were considered for this analysis: 63,579 trees from at least 82 genera. Figure E2 shows the spatial distribution of trees superimposed over an NDVI map.

We used two definitions to classify tree genera into allergenic and non-allergenic. Both definitions are based on the same three criteria. For a tree genus to be classified as allergenic according to definition 1, it had to satisfy two out of the three criteria. The stricter definition 2 required that all three criteria be satisfied. The first criterion is satisfied by tree genera whose pollen are being routinely monitored in Germany (http://www.pollenstiftung.de/pollenvorhersage/pollenflug-kalender). The second and third criteria relate to tree species and are satisfied by a genus if among the trees of that genus that were planted in Leipzig before 1998 there is at least one species that satisfies the criterion. The second criterion is satisfied by a 
species if its pollen allergens were characterized in a published study, or if it was described by a study as causing allergenic reactions in people that came into contact with its pollen (e.g. measured by skin prick test), or if there are reports about its sensitization/allergenicity rates. Literature search was done via Google Scholar in combination with the library access service of Technical University Munich (TUM OPAC plus). The third criterion is satisfied by a species if www.allergen.org, the most complete database of allergens, had at least one aeroallergen listed for it as of September 2, 2019. The details on all tree genera of the city of Leipzig planted before 1998, together with their abundances, are provided in Table E1. According to definition 1, 21,324 trees from the following 16 genera (sorted by tree count in descending order) were classified as allergenic: Fraxinus (ash), Quercus (oak), Platanus(plane), Prunus (prunus), Betula (birch), Populus(poplar), Carpinus (hornbeam), Corylus (hazel), Salix (willow), Pinus (pine), Fagus (beech), Alnus(alder), Sambucus (elder), Castanea (chestnut), Syringa (lilac) and Juniperus (juniper). According to definition 2, there were 9,453 allergenic trees from 7 genera: Fraxinus, Betula, Carpinus, Corylus, Fagus, Alnus and Syringa .

Mean NDVI, total number of trees and number of allergenic trees according to the two definitions were calculated for circular buffers of 100, 500 and 1,000 m around each participant's home address at birth.

Geographic data pre-processing and calculations were conducted with ArcMap 10.4 and ArcGIS Pro 2.2 Geographical Information System (ESRI, Redlands, CA, USA).

\section{Air pollution}

The annual average concentrations of air pollutants at residential addresses at birth were assigned from raster surfaces at a resolution of $100 \times 100 \mathrm{~m}$. Estimates for nitrogen dioxide $\left(\mathrm{NO}_{2}\right)$ and warm season ozone were available for the year 2000 from the ELAPSE project. ${ }^{20}$ Both air pollutants were modelled by hybrid land use regression models using data from AirBase, a database of routine air pollution measurements in the EU member states. The hybrid models for western Europe that used dispersion model estimates, land use and road data and for ozone also kriging on the residuals, were able to explain $60 \%$ and $82 \%$ of the variation in measured $\mathrm{NO}_{2}$ and ozone concentrations, respectively.

\section{Statistical analysis}

Given the repeated assessment of allergic outcomes, generalized estimating equations (GEE) with logit link and exchangeable correlation structure were used to regress the prevalence of allergic outcomes up to 15 years on the exposures of interest at birth.

First, we used generalized additive models (GAM) ${ }^{21}$ to test the non-linearity of the univariate relationships between each pair of exposure and "ever" outcome (e.g., ever-asthma is a constructed outcome variable that is 1 if at least one of the age-specific asthma variables is 1 and otherwise 0 ). We discovered that associations deviated from linearity in many cases. Therefore, we categorized NDVI variables and tree counts for the 500 $\mathrm{m}$ and $1000 \mathrm{~m}$ buffers into tertiles. Tree counts for the $100 \mathrm{~m}$ buffer were dichotomized (=0vs [?] 1).

In line with our previous analyses on greenspace and allergic outcomes, ${ }^{11,12}$ we considered the $500 \mathrm{~m}$ buffer variables our main exposures of interest. The main models were a prioriadjusted for age, sex, family history of allergic diseases, parental education (based on the highest number of years of school education of either parent: $<10$ years, $=10$ years, $>10$ years, according to the German educational system) and season of birth (October to January, February to March, May to July, August to September, corresponding to next to no pollen, tree pollen season, grass pollen season, ragweed and mugwort pollen season, respectively). Season of birth was categorized according to a regional pollen calendar (http://www.pollenstiftung.de/pollenvorhersage/pollenflug-kalender).

A series of sensitivity analyses were performed. This included crude analyses, which were adjusted only for age, and additionally adjusted analyses with main models also controlled for maternal smoking during pregnancy and tobacco smoke exposure at home until age 4 (yes, likely no, no), presence of older siblings, exclusive breastfeeding during the first four months and birth weight (grams). We also checked the robustness of the results by using $100 \mathrm{~m}$ and 1,000 m buffers. Moreover, the models for total tree count and allergenic 
tree count according to definitions 1 and 2 were additionally adjusted for NDVI to account for vegetation not captured by the tree registry, e.g., trees on private grounds, herbs, bushes. Furthermore, we reran the analyses excluding participants with partially missing outcome data. Effect modification by age was tested by introducing an interaction term between the exposure variable and age. Additionally, we stratified our analyses by whether participants changed their place of residence between birth and 2 years of age. Finally, we checked effect modification by whether participants resided within $300 \mathrm{~m}$ from the nearest urban green space or forest of at least 1 ha ${ }^{22}$. Green spaces were derived using the Urban Atlas land use data for the year 2006 (http://www.eea.europa.eu/data-and-maps/data/urban-atlas).

To check whether air pollution modifies the association of interest, an interaction term between the air pollutant and the exposure variable was introduced. Additionally, models were stratified by tertiles of $\mathrm{NO}_{2}$ and summer ozone levels. In all stratified analyses, we combined low and medium categories of parental education, as there were sometimes too few cases in the lowest educational category.

Data pre-processing and statistical analyses were done using the statistical software R 3.6.1 (Vienna, Austria). ${ }^{23}$ GEE models were fitted by the geeglm() function from the geepack package. ${ }^{24}$ GAM models were executed using the $g a m()$ function from the $m g c v$ package. ${ }^{25}$

\section{Results}

\section{Descriptive characteristics of the study population and exposure variables}

Over half of the children $(55.2 \%)$ were from families with high level of parental education while only $2.4 \%$ were from families with low level of parental education (Table 1). This was the only systematic difference between the analytic sample compared to the original LISA Leipzig population where the above-mentioned numbers were $48 \%$ and $5 \%$, respectively.

The highest prevalence of doctor diagnosed asthma (4.5\%) was observed at 7 and at 11 years (Table 2). The highest prevalence of AR $(12.2 \%)$ was observed at 11 years. $47.4 \%$ children at 15 years were sensitized to aeroallergens. The peak of sensitization to food allergens $(16.5 \%)$ was reached at 10 years.

Descriptions of the exposure variable categories for all three buffer sizes are provided in Table E2. Mean and standard deviations for $\mathrm{NO}_{2}$ and summer ozone levels were $33.5 \pm 4.5 \mu \mathrm{g} / \mathrm{m}^{3}$ and $84.7 \pm 3.9 \mu \mathrm{g} / \mathrm{m}^{3}$, respectively.

\section{Greenness, trees and allergenic trees and allergic outcomes: main results}

Birth home addresses surrounded by medium greenness (second NDVI tertile) in a $500 \mathrm{~m}$ buffer were associated with increased prevalence of asthma up to 15 years (odds ratio $(\mathrm{OR})=1.46$ ) (Table 3). Home addresses with high greenness (third NDVI tertile) showed decreased prevalence of asthma $(\mathrm{OR}=0.44)$. Medium numbers of allergenic trees (second tertile) according to definition 1 were related to higher prevalence of asthma $(\mathrm{OR}=1.92)$. This was not the case for definition 2. Medium and high numbers of trees and allergenic trees according to the both definitions were associated with higher prevalence of AR (Table 3). All exposures were related to higher allergic sensitization to aeroallergens, and high numbers of allergenic trees according to definition 1 were related to food sensitization.

\section{Greenness, trees, allergenic trees and allergic outcomes: results from sensitivity analyses}

Effect estimates were similar when we adjusted the three models with tree exposure variables additionally for NDVI (Table 3). Similar associations were observed for the $100 \mathrm{~m}$ (Table E3) and 1,000 m (Table E4) buffers, with some exceptions. In case of the $100 \mathrm{~m}$ buffer, associations between NDVI and asthma, the association between allergenic trees definition 1 and food sensitization, and the associations of total number of trees and NDVI with aeroallergen sensitization were not present. For the 1,000 m buffer, no associations with aeroallergen sensitization were observed and the association between medium NDVI and asthma was lost.

Effect estimates from crude and additionally adjusted models were very similar to those from the main models (Table E5). The same was true when children with partially missing outcome variables were excluded (data 
not shown). The associations between trees and asthma, allergenic trees definition 2 and asthma, and NDVI and aeroallergen sensitization got stronger with age. Effect modification by moving between birth and 2 years did not reveal a distinct pattern (Figure E3). Similarly, a mixed pattern was observed considering effect modification by residing close to structured green spaces (Figure E4).

\section{Effect modification by air pollutants}

The associations of trees and allergenic trees definition 1 with higher AR and asthma were stronger in the high tertile of $\mathrm{NO}_{2}$ compared to the low tertile of $\mathrm{NO}_{2}$ (Figure 1). The same holds for both definitions of allergenic trees and sensitization to aeroallergens. The associations of trees with aeroallergen sensitization and of allergenic trees definition 2 and food sensitization showed the opposite tendency. The associations of trees and allergenic trees and AR were stronger for the low ozone tertile compared to the high ozone tertile, the same was observed for the association between allergenic trees and aeroallergen sensitization (Figure E5).

\section{Discussion}

The results of our longitudinal analyses in 631 children from the city of Leipzig, Germany, show that early life residence in places with many trees and allergenic trees specifically, was consistently associated with increased prevalence of AR up to 15 years and, to a lesser extent, also with increased prevalence of allergic sensitization to aeroallergens. Associations with asthma and sensitization to food allergens were inconsistent. Associations with greenness, as measured by NDVI, were either inconclusive (for asthma) or mostly nonsignificant (for other outcomes). Effect estimates for trees and allergenic trees and AR tended to be stronger in high $\mathrm{NO}_{2}$ and low ozone settings.

\section{Comparison with previous findings and interpretations}

We are not aware of previous studies on long-term exposure to allergenic trees and allergic outcomes. Many studies, however, considered NDVI in relation to asthma, AR and sometimes to allergic sensitization to aeroallergens. There are also several studies on trees and allergic outcomes.

\section{Residential greenness and allergic outcomes}

In line with our observations, the reported associations for NDVI and asthma were heterogeneous and, in some cases, non-linear. Like in our study, medium but not high NDVI increased the risk of asthma in 7,040 Taiwanese children. ${ }^{26}$ Also in line with us, exposure to medium but not high greenness was associated with asthma in the Euro-Siberian climatic region of a Spanish multicentre study of 2,472 children. ${ }^{27}$ Nevertheless, high greenness was protective against wheezing in the same region of the same study. ${ }^{27}$ Higher NDVI in the $100 \mathrm{~m}$ buffer, but not in larger buffers, was related to increased asthma risk in 1,489 Kaunas children, and this association was stronger in children living further away from parks. ${ }^{28}$ We observed opposite tendencies. Like our protective association between the high NDVI tertile and asthma, two other urban studies - one in 1,915 Chicago children ${ }^{29}$ and another one in 187 Turin children $^{30}$ - reported protective greenness effects on asthma. Higher greenness was also related to lower asthma risk in a large New Zealand study of 49,956 children. ${ }^{31}$ Nevertheless, an even larger study $(\mathrm{n}=65,000)$ from the Vancouver Metropolitan Area failed to detect such an association. ${ }^{32}$ Null findings between NDVI and asthma were also reported in 3,178 Spanish children $^{33}$ and 5,643 children from Suzhou, China. ${ }^{34}$

We did not observe any association between NDVI and AR and the association between NDVI and aeroallergen sensitization was inconsistent. This is in line with the aforementioned studies by Tischer et al., ${ }^{27}$ Dadvand et al. ${ }^{33}$ and $\mathrm{Li}$ et al. ${ }^{34}$ In addition, Gernes et al. ${ }^{35}$ reported null findings between NDVI and rhinitis and atopic sensitization in 425 children from the USA. Our two previous multi-centre studies, whose study populations were distinct from the one used in this analysis, reported differential associations with $\mathrm{AR}$, nose and eye symptoms and aeroallergen sensitization, as is mentioned in the Introduction. ${ }^{11,12}$ Interestingly, effect modification by moving did not reveal any consistent trends in Fuertes et al., ${ }^{12}$ in line with our present analysis. Unlike our stratified results by air pollutants, no pattern was observed in analyses stratified by $\mathrm{NO}_{2}$ subgroups in these two studies. ${ }^{11,12}$ 


\section{Residential trees and allergic outcomes}

Other studies on trees and allergic outcomes did not observe an association with AR. ${ }^{35,36}$ This contrasts with our analysis where this association was the strongest and the most consistent. Yet, Lovasi et al. ${ }^{36}$ reported increased risks of asthma and allergic sensitization in 549 7-year-old residents of New York, which was partially in line with us. Both studies used tree cover extracted from aerial images. Tree cover does not capture differences between trees.

We used data from a tree registry to distinguish between allergenic and non-allergenic trees. Our results for AR and to a lesser extent for aeroallergen sensitization suggest that in Leipzig having any trees close to home has about the same negative health effect as having allergenic trees close by. This might not be the case for other study areas. Future research might show that comparisons of findings concerning trees and allergic outcomes are only valid between cities with similar tree patterns, air quality and urban climate. ${ }^{37}$

No epidemiological study so far had enough information to adequately deal with the problem that pollen loads as well as allergenic potentials may vary between tree individuals and for the same tree individuum, also across years. ${ }^{38}$ For most allergenic tree species this phenomenon has not yet been explored let alone been understood.

\section{Effect modification by air pollutants}

Trees and allergenic trees were associated with a higher prevalence of $\mathrm{AR}$ when $\mathrm{NO}_{2}$ levels were comparatively high or ozone was comparatively low. Apart from the direct detrimental effect of nitric oxides on respiratory health, ${ }^{39,40}$ these substances may have varying effects on the allergenicity and amount of pollen depending on plant species and environmental conditions. For example, a number of molecular studies reviewed by Frank and Ernst ${ }^{41}$ found increasing allergenicity (tested by increased $\mathrm{IgE}$ reactivity) of $\mathrm{NO}_{2}$-treated pollen of the common species Acer negundo , Betula pendula , Ostrya carpinifolia and Carpinus betulus , ${ }^{42,43}$ while Beck et al..$^{13}$ did not find a correlation of birch (Betula pendula ) pollen allergenicity with $\mathrm{NO}_{2}$ levels. Divergent findings were also observed for ozone treatments. ${ }^{41}$ In addition, $\mathrm{NO}_{2}$ and ozone may have different effects on pollen allergenicity even within the same species. ${ }^{44}$ The effect of these pollutants on a larger scale is further blurred by the chemical reactions between ozone and $\mathrm{NO}_{2}$ that depend atmospheric conditions. ${ }^{45,46}$ This makes it difficult for epidemiological studies like ours to investigate their influence on the associations of trees and allergenic trees with allergic outcomes.

\section{Implications for city greening}

Cities and their rural surroundings are often quite different environments in terms of their tree composition. For Leipzig, the overlap between tree genera planted in the city and those growing in regional forests is small. Unlike Leipzig, the surrounding forests consist mostly of spruce and pine (https://bwi.info, data for Saxony, accessed 2020-03-07), that is, taxa of no or minor importance as allergen producers (cf. Table E1). By planting trees in proportions found in natural habitats and eliminating highly allergenic taxa ${ }^{47,48}$ city greening may become even more beneficial.

Attention to allergenic potential is also important when selecting tree species that are able to withstand the challenges posed by climate change. Resilient tree taxa ${ }^{49-51}$ may prove detrimental for the development of allergenic diseases. Examples includeAlnus $x$ spaethii ${ }^{52}$ and Corylus colurna,a close relative of the major allergen source Corylus avellana,which despite their sturdiness should be reconsidered as future city trees.

\section{Implications for future research}

We argue that NDVI and other vegetation indices are not just unable to differentiate vegetation types but are also easily confounded by urbanicity and thus air pollution. When investigating associations where knowing the amount of greenspace or the distance to greenspace is not enough, like with allergic outcomes, we suggest that more detailed vegetation measures be considered. In our study, number of trees appeared to be a good proxy for number of allergenic trees, but this unlikely to always be the case across study areas. Digital tree registry data are scarce but available for some cities, including New York, Frankfurt am Main, 
Vienna, Hamburg and Melbourne. Similarly, rich datasets are available for different areas, like the maps of 12 key allergenic taxa in the UK for the year 2012 in $1 \times 1 \mathrm{~km}$ resolution produced by McInnes et al. ${ }^{53}$

\section{Strengths and limitations}

Besides the modest sample size and somewhat limited external validity due to loss to follow-up, primarily of families with low school education, the largest limitation of our study is that it is based on a single study area. Therefore, our findings cannot be generalized to other areas. Future studies should further investigate our observed associations in urban, suburban and rural contexts across different climates and countries. Moreover, we cannot exclude residual confounding of our findings by allergenic shrubs and herbs and even by trees on private grounds, as these were not part of the tree registry. We co-adjusted our models with tree exposures for general vegetation degree, but this is not sufficient to rule out potential impacts of other allergenic vegetation. Limited sample size together with relatively low prevalence of asthma and AR precluded us from testing more effect modifiers, in particular, socioeconomic status. Nevertheless, LISA is an established cohort study meant to explore various risk factors of allergic diseases. All allergic outcomes were collected by using state-of-the-art methods at many timepoints. Repeated measurements analysis provides enough power to detect even small associations. The impact of confounding was reduced by controlling for many perinatal and early postnatal allergy risk factors. Finally, we were able to classify all tree genera into allergenic vsnon-allergenic by several different criteria, something that was never done before in epidemiological analyses.

\section{Conclusions}

We observed that early life residence in places with many trees and specifically many allergenic trees may increase prevalence of AR later in life regardless of general level of vegetation. This association, as well as its modification by nitrogen dioxide and ozone, needs replication in further studies.

\section{Conflict of interest}

The authors declare that they have no conflict of interest.

\section{Acknowledgements}

We thank all children and parents for their cooperation, and all technical and administrative support staff and medical and field work teams. We are also grateful to all members of the LISA Study Group. The LISA Study group consists of the following: Helmholtz Zentrum München, German Research Center for Environmental Health, Institute of Epidemiology, Munich (Heinrich J, Schnappinger M, Brüske I, Ferland M, Schulz H, Zeller C, Standl M, Thiering E, Tiesler C, Flexeder C); Department of Pediatrics, Municipal Hospital "St. Georg", Leipzig (Borte M, Diez U, Dorn C, Braun E); Marien Hospital Wesel, Department of Pediatrics, Wesel (von Berg A, Berdel D, Stiers G, Maas B); Pediatric Practice, Bad Honnef (Schaaf B); Helmholtz Centre of Environmental Research - UFZ, Department of Environmental Immunology, Leipzig (Lehmann I, Bauer M, Röder S, Schilde M, Nowak M, Herberth G, Müller J); Technical University Munich, Department of Pediatrics, Munich (Hoffmann U, Paschke M, Marra S); Clinical Research Group Molecular Dermatology, Department of Dermatology and Allergy, Technische Universität München (TUM), Munich (Ollert M, Grosch J).

We would also like to thank Sebastian Fried, Sachgebiet Stadtbäume, Amt für Stadtgrün und Gewässer, Stadt Leipzig, Germany, for providing us Leipzig tree registry ("Baumkataster") data without which this analysis would not have been possible.

\section{Author's contributions}

IM and JH conceived the study. RM and FW classified Leipzig trees into allergenic and non-allergenic. KdH developed air pollution grids. IM assigned all GIS variables to participants, analysed the data and wrote the first draft of the manuscript. FW contributed to drafting the first version of the manuscript. CB assisted with the data analysis. JH, GH and MS have made substantial contributions to the conception, design and 
acquisition of the LISA Leipzig data. All authors involved in the result interpretation and text revision. All authors approved the final manuscript.

\section{References}

1. Houlden V, Weich S, Porto de Albuquerque J, Jarvis S, Rees K. The relationship between greenspace and the mental wellbeing of adults: A systematic review. PLoS One. 2018;13(9):e0203000.

2. Rojas-Rueda D, Nieuwenhuijsen MJ, Gascon M, Perez-Leon D, Mudu P. Green spaces and mortality: a systematic review and meta-analysis of cohort studies. Lancet Planet Health. 2019;3(11):e469-e477.

3. den Braver NR, Lakerveld J, Rutters F, Schoonmade LJ, Brug J, Beulens JWJ. Built environmental characteristics and diabetes: a systematic review and meta-analysis. BMC Med. 2018;16(1):12.

4. Twohig-Bennett C, Jones A. The health benefits of the great outdoors: A systematic review and metaanalysis of greenspace exposure and health outcomes. Environ Res. 2018;166:628-637.

5. Markevych I, Schoierer J, Hartig T, Chudnovsky A, Hystad P, Dzhambov AM, et al. Exploring pathways linking greenspace to health: Theoretical and methodological guidance. Environ Res. 2017;158:301-317.

6. von Mutius E, Vercelli D. Farm living: effects on childhood asthma and allergy. Nat Rev. 2010;10:861868.

7. Rook GA. Regulation of the immune system by biodiversity from the natural environment: an ecosystem service essential to health. Proc Natl Acad Sci U S A. 2013;110(46):18360-18367.

8. Erbas B, Jazayeri M, Lambert KA, Katelaris CH, Prendergast LA, Tham R, et al. Outdoor pollen is a trigger of child and adolescent asthma emergency department presentations: A systematic review and meta-analysis. Allergy. 2018;73(8):1632-1641.

9. Lambert KA, Bowatte G, Tham R, Lodge C, Prendergast L, Heinrich J, et al. Residential greenness and allergic respiratory diseases in children and adolescents - A systematic review and meta-analysis. Environ Res. 2017;159:212-221.

10. Lambert KA, Bowatte G, Tham R, Lodge CJ, Prendergast LA, Heinrich J, et al. Greenspace and Atopic Sensitization in Children and Adolescents-A Systematic Review. Int J Environ Res Public Health. 2018;15(11):2539.

11. Fuertes E, Markevych I, von Berg A, Bauer CP, Berdel D, Koletzko S, et al. Greenness and allergies: evidence of differential associations in two areas in Germany. J Epidemiol Community Health. 2014;68(8):787-790.

12. Fuertes E, Markevych I, Bowatte G, Gruzieva O, Gehring U, Becker A, et al. Residential greenness is differentially associated with childhood allergic rhinitis and aeroallergen sensitization in seven birth cohorts. Allergy 2016;71(10):1461-1471.

13. Beck I, Jochner S, Gilles S, McIntyre M, Buters JT, Schmidt-Weber C, et al. High environmental ozone levels lead to enhanced allergenicity of birch pollen. PLoS One. 2013;8(11):e80147.

14. Eisenman TS, Churkina G, Jariwala SP, Kumar P, Lovasi G, Pataki DE, et al. Urban trees, air quality, and asthma: An interdisciplinary review. Landscape and Urban Planning 2019;187:47-59.

15. Heinrich J, Bruske I, Cramer C, Hoffmann U, Schnappinger M, Schaaf B, et al. GINIplus and LISAplus - Design and selected results of two German birth cohorts about natural course of atopic diseases and their determinants. Allergol Select. 2017;1(1):85-95.

16. Zutavern A, Brockow I, Schaaf B, Bolte G, von Berg A, Diez U, et al. Timing of solid food introduction in relation to atopic dermatitis and atopic sensitization: results from a prospective birth cohort study. Pediatrics 2006;117:401-411.

17. Markevych I, Standl M, Lehmann I, von Berg A, Heinrich J. Food diversity during the first year of life and allergic diseases until 15 years. J Allergy Clin Immunol 2017c;140(6):1751-1754.e4.

18. Tucker CJ. Red and Photographic Infrared Linear Combinations for Monitoring Vegetation. Remote Sensing of Environment 1979;8(2):127-150.

19. Dadvand P, Sunyer J, Basagana X, Ballester F, Lertxundi A, Fernandez-Somoano A, et al. Surrounding greenness and pregnancy outcomes in four Spanish birth cohorts. Environ Health Perspect. $2012 ; 120(10): 1481-1487$. 
20. de Hoogh K, Chen J, Gulliver J, Hoffmann B, Hertel O, Ketzel M, et al. Spatial $\mathrm{PM}_{2.5}, \mathrm{NO}_{2}, \mathrm{O}_{3}$ and BC models for Western Europe - Evaluation of spatiotemporal stability. Environ Int. 2018;120:81-92.

21. Hastie T, Tibshirani R. Generalized additive models. Stat Sc. 1986;1(3):297-318.

22. Annerstedt van den Bosch M, Mudu P, Uscila V, Barrdahl M, Kulinkina A, Staatsen B, et al. Development of an urban green space indicator and the public health rationale. Scand J Public Health 2016;44(2):159-67.

23. R Core Team: R: A Language and Environment for Statistical Computing. Vienna, Austria: R Foundation for Statistical Computing; 2012. [http://www.R-project.org/].

24. Hojsgaard S, Halekoh U, Yan J. The R Package geepack for Generalized Estimating Equations. Journal of Statistical Software 2006;15(2):1-11.

25. Wood SN. Fast stable restricted maximum likelihood and marginal likelihood estimation of semiparametric generalized linear models. Journal of the Royal Statistical Society (B) 2011;73(1):3-36.

26. Hsieh CJ, Yu PY, Tai CJ, Jan RH, Wen TH, Lin SW, et al. Association between the First Occurrence of Asthma and Residential Greenness in Children and Teenagers in Taiwan. Int J Environ Res Public Health. 2019;16(12):2076.

27. Tischer C, Gascon M, Fernandez-Somoano A, Tardon A, Lertxundi Materola A, Ibarluzea J, et al. Urban green and grey space in relation to respiratory health in children. Eur Respir J. 2017:49.

28. Andrusaityte S, Grazuleviciene R, Kudzyte J, Bernotiene A, Dedele A, Nieuwenhuijsen MJ. Associations between neighbourhood greenness and asthma in preschool children in Kaunas, Lithuania: a case-control study. BMJ Open. 2016;6:e010341.

29. Eldeirawi K, Kunzweiler C, Zenk S, Finn P, Nyenhuis S, Rosenberg N, et al. Associations of urban greenness with asthma and respiratory symptoms in Mexican American children. Ann Allergy Asthma Immunol. 2019;122(3):289-295.

30. Squillacioti G, Bellisario V, Levra S, Piccioni P, Bono R. Greenness Availability and Respiratory Health in a Population of Urbanised Children in North-Western Italy. Int J Environ Res Public Health. 2019;17(1):108.

31. Donovan GH, Gatziolis D, Longley I, Douwes J. Vegetation diversity protects against childhood asthma: results from a large New Zealand birth cohort. Nat Plants. 2018;4(6):358-364.

32. Sbihi H, Koehoorn M, Tamburic L, Brauer M. Asthma Trajectories in a Population-based Birth Cohort. Impacts of Air Pollution and Greenness. Am J Respir Crit Care Med. 2017;195(5):607-613.

33. Dadvand P, Villanueva CM, Font-Ribera L, Martinez D, Basagana X, Belmonte J, et al. Risks and benefits of green spaces for children: a cross-sectional study of associations with sedentary behavior, obesity, asthma, and allergy. Environ Health Perspect. 2014;122(12):1329-35.

34. Li L, Hart JE, Coull BA, Cao SJ, Spengler JD, Adamkiewicz G. Effect of Residential Greenness and Nearby Parks on Respiratory and Allergic Diseases among Middle School Adolescents in a Chinese City. Int J Environ Res Public Health. 2019;16(6):991.

35. Gernes R, Brokamp C, Rice GE, Wright JM, Kondo MC, Michael YL, et al. Using high-resolution residential greenspace measures in an urban environment to assess risks of allergy outcomes in children. Sci Total Environ. 2019;668:760-767.

36. Lovasi GS, O'Neil-Dunne JP, Lu JW, Sheehan D, Perzanowski MS, Macfaden SW, et al. Urban tree canopy and asthma, wheeze, rhinitis, and allergic sensitization to tree pollen in a New York City birth cohort. Environ Health Perspect. 2013;121(4):494-500.

37. de Weger LA, Bergmann KC, Rantio-Lehtimaki A, Dahl A, Buters J, Dechamp C, et al. Impact of pollen. In: Sofiev M., Bergmann KC. (Eds.). Allergenic Pollen. Dordrecht Springer, 2013: 161-215.

38. Buters JT, Kasche A, Weichenmeier I, Schober W, Klaus S, Traidl-Hoffmann C, et al. Year-to-year variation in release of Bet $\mathrm{v} 1$ allergen from birch pollen: evidence for geographical differences between West and South Germany. Int Arch Allergy Immunol. 2008;145(2):122-30.

39. Orellano P, Quaranta N, Reynoso J, Balbi B, Vasquez J. Effect of outdoor air pollution on asthma exacerbations in children and adults: Systematic review and multilevel meta-analysis. PLoS One. 2017;12(3):e0174050.

40. Li S, Williams G, Jalaludin B, Baker P. Panel studies of air pollution on children's lung function and 
respiratory symptoms: a literature review. J Asthma. 2012;49(9):895-910.

41. Frank U, Ernst D. Effects of $\mathrm{NO}_{2}$ and Ozone on Pollen Allergenicity. Front Plant Sci. 2016;7:91.

42. Cuinica LG, Abreu I, da Silva J. Effect of air pollutant NO2 on Betula pendula, Ostrya carpinifolia and Carpinus betulus pollen fertility and human allergenicity. Environ Pollut 2014;186:50-55.

43. Sousa R, Duque L, Duarte AJ, Gomes CR, Ribeiro H, Cruz A, et al. In vitro exposure of Acer negundo pollen to atmospheric levels of $\mathrm{SO} 2$ and NO2: effects on allergenicity and germination. Environ Sci Technol 2012;46:2406-2412.

44. Zhao F, Durner J, Winkler JB, Traidl-Hoffmann C, Strom TM, Ernst D, et al. Pollen of common ragweed (Ambrosia artemisiifolia L.): Illumina-based de novo sequencing and differential transcript expression upon elevated $\mathrm{NO}_{2} / \mathrm{O}_{3}$. Environ Pollut. 2017;224:503-514.

45. Murphy JG, Day DA, Cleary PA, Wooldridge PJ, Millet DB, Goldstein AH, et al. The weekend effect within and downwind of Sacramento? Part 1: Observations of ozone, nitrogen oxides, and VOC reactivity. Atmos Chem Phys. 2007;7(20):5327-5339.

46. Roberts-Semple D, Song F, Gao Y. Seasonal characteristics of ambient nitrogen oxides and ground-level ozone in metropolitan northeastern New Jersey. Atmospheric Pollution Research 2012;3:247-257.

47. Bergmann CH, Zuberbier T, Augustin J, Mucke HG, Straff W. Climate change and pollen allergy: cities and municipalities should take people suffering from pollen allergy into account when planting in public spaces. Allergo J. 2012;21(2):103-107.

48. Carinanos P, Casares-Porcel M. Urban green zones and related pollen allergy: A review. Some guidelines for designing spaces with low allergy impact. Landscape \& Urban Planning 2011;101(3):205-214.

49. Roloff A, Korn S, Gilner S. The Climate-Species-Matrix to select tree species for urban habitats considering climate change. Urban Forestry \& Urban Greening. 2009;8:295-308.

50. Gillner S, Vogt J, Tharang A, Roloff A. Role of street trees in mitigating effects of heat and drought at highly sealed urban sites. Landscape \& Urban Planning. 2015;143:33-42.

51. McPherson EG, Berry AM, can Doorn NS. Performance testing to identify climate-ready trees. Urban Forestry \& Urban Greening. 2018;29:28-39.

52. Gehrig R, Gassner M, Schmid-Grendmeier P. Alnus x spaethii pollen can cause allergies already at Christmas. Aerobiologia. 2015;31:239-247.

53. McInnes RN, Hemming D, Burgess P, Lyndsay D, Osborne NJ, Skjoth CA, et al. Mapping allergenic pollen vegetation in UK to study environmental exposure and human health. Sci Total Environ. 2017;599-600:483-499.

Table 1. Descriptive characteristics of the analytic sample

\begin{tabular}{llll}
\hline Characteristic & Category & $\mathbf{n} / \mathbf{N}$ & $\mathbf{\%}$ \\
\hline Season of birth & October to January & $224 / 631$ & 35.5 \\
& February to April & $169 / 631$ & 26.8 \\
& May to July & $145 / 631$ & 23.0 \\
Sex & August to September & $93 / 631$ & 14.7 \\
& Female & $324 / 631$ & 51.3 \\
Highest parental education & Male & $307 / 631$ & 48.7 \\
& Low (<10 years) & $15 / 631$ & 2.4 \\
Parental atopy & Medium (=10 years) & $268 / 631$ & 42.5 \\
& High (>10 years) & $348 / 631$ & 55.2 \\
Older siblings & No & $342 / 631$ & 54.2 \\
& Yes & $289 / 631$ & 45.8 \\
Smoking during pregnancy & No & $416 / 631$ & 65.9 \\
& Yes & $215 / 631$ & 34.1 \\
Smoking at home between birth and 4 years & No & $514 / 612$ & 84.0 \\
& Yes & $98 / 612$ & 16.0 \\
& Never & $318 / 629$ & 50.6
\end{tabular}




\begin{tabular}{llll}
\hline Characteristic & Category & n/N & \% \\
\hline & Ever & $255 / 629$ & 40.5 \\
Exclusive breastfeeding first 4 months & No & $262 / 619$ & 42.3 \\
& Yes & $357 / 619$ & 57.7 \\
Birth weight (grams) & Mean and SD & 3509.0 & 453.9 \\
Changed place of residence between birth and 2 years & No & $471 / 631$ & 74.6 \\
& Yes & $160 / 631$ & 25.4 \\
\hline
\end{tabular}

$\mathrm{SD}$ - standard deviation; $\mathrm{n}$ - number of participants in the category; $\mathrm{N}$ - number of participants with available data.

Table 2. Prevalence of allergic outcomes in the analytic sample at all ages

\begin{tabular}{lllllll}
\hline Age, years & Asthma & Asthma & Allergic rhinitis & Allergic rhinitis & Aeroallergen sensitization & Aeroallerg \\
\hline & $\mathbf{n} / \mathbf{N}$ & $\mathbf{\%}$ & $\mathbf{n} / \mathbf{N}$ & $\mathbf{0}$ & $\mathbf{n} / \mathbf{N}$ & $\mathbf{2}$ \\
2 & & & & & & 4.9 \\
3 & $3 / 534$ & 0.6 & $8 / 535$ & 1.5 & & \\
4 & $7 / 524$ & 1.3 & $13 / 520$ & 2.5 & & \\
5 & $11 / 459$ & 2.4 & $19 / 462$ & 4.1 & $76 / 262$ & \\
6 & $12 / 461$ & 2.6 & $29 / 461$ & 6.3 & & \\
7 & $16 / 356$ & 4.5 & $24 / 353$ & 6.8 & & \\
8 & $11 / 356$ & 3.1 & $27 / 355$ & 7.6 & & \\
9 & $13 / 356$ & 3.7 & $31 / 354$ & 8.8 & & \\
10 & $10 / 356$ & 2.8 & $40 / 353$ & 11.3 & & \\
11 & $16 / 355$ & 4.5 & $43 / 353$ & 12.2 & & \\
12 & $12 / 355$ & 3.4 & $28 / 351$ & 8.0 & & \\
13 & $14 / 355$ & 3.9 & $29 / 351$ & 8.3 & & \\
14 & $11 / 355$ & 3.1 & $31 / 351$ & 8.8 & & \\
15 & $10 / 355$ & 2.8 & $30 / 351$ & 8.5 & & \\
\hline
\end{tabular}

$\mathrm{n}$ - number of participants in the category; $\mathrm{N}$ - number of participants with available data.

Table 3. Associations between greenness, trees and allergenic trees in $500 \mathrm{~m}$ buffers around birth residential addresses with allergic outcomes up to 15 years, estimated by GEE models

\begin{tabular}{|c|c|c|c|c|c|}
\hline & Exposure & Asthma & Asthma & Asthma & Allergic $\mathrm{rh}$ \\
\hline \multirow{13}{*}{ Single exposure models } & & OR & LCI & UCI & OR \\
\hline & NDVI 500 m (T1 ref.) & & & & \\
\hline & $\mathrm{T} 2$ & 1.46 & 1.02 & 2.10 & 1.26 \\
\hline & $\mathrm{T} 3$ & 0.44 & 0.27 & 0.72 & 0.90 \\
\hline & Trees 500 m (T1 ref.) & & & & \\
\hline & $\mathrm{T} 2$ & 1.06 & 0.72 & 1.55 & 1.62 \\
\hline & $\mathrm{T} 3$ & 0.77 & 0.50 & 1.17 & 2.13 \\
\hline & Allergenic trees (def. 1) $500 \mathrm{~m}$ (T1 ref.) & & & & \\
\hline & $\mathrm{T} 2$ & 1.92 & 1.29 & 2.84 & 2.09 \\
\hline & $\mathrm{T} 3$ & 0.99 & 0.63 & 1.58 & 1.70 \\
\hline & Allergenic trees (def. 2) $500 \mathrm{~m}$ (T1 ref.) & & & & \\
\hline & $\mathrm{T} 2$ & 1.39 & 0.94 & 2.06 & 2.06 \\
\hline & $\mathrm{T} 3$ & 0.89 & 0.58 & 1.38 & 2.06 \\
\hline
\end{tabular}




\begin{tabular}{|c|c|c|c|c|c|}
\hline & Exposure & Asthma & Asthma & Asthma & Allergic rh \\
\hline \multirow[t]{18}{*}{ Models adjusted for NDVI } & Trees 500 m (T1 ref.) & & & & \\
\hline & $\mathrm{T} 2$ & 0.78 & 0.53 & 1.15 & 1.62 \\
\hline & $\mathrm{T} 3$ & 0.54 & 0.35 & 0.84 & 2.13 \\
\hline & NDVI 500 m (T1 ref.) & & & & \\
\hline & $\mathrm{T} 2$ & 1.44 & 1.01 & 2.07 & 1.30 \\
\hline & T3 & 0.37 & 0.23 & 0.61 & 1.12 \\
\hline & Allergenic trees (def. 1) $500 \mathrm{~m}$ (T1 ref.) & & & & \\
\hline & $\mathrm{T} 2$ & 1.49 & 0.97 & 2.30 & 2.03 \\
\hline & $\mathrm{T} 3$ & 0.82 & 0.50 & 1.33 & 1.68 \\
\hline & NDVI 500 m (T1 ref.) & & & & \\
\hline & $\mathrm{T} 2$ & 1.38 & 0.96 & 1.97 & 1.23 \\
\hline & $\mathrm{T} 3$ & 0.45 & 0.27 & 0.74 & 1.03 \\
\hline & Allergenic trees (def. 2) $500 \mathrm{~m}$ (T1 ref.) & & & & \\
\hline & $\mathrm{T} 2$ & 1.29 & 0.86 & 1.93 & 2.03 \\
\hline & $\mathrm{T} 3$ & 0.72 & 0.45 & 1.15 & 1.98 \\
\hline & NDVI 500 m (T1 ref.) & & & & \\
\hline & $\mathrm{T} 2$ & 1.46 & 1.00 & 2.12 & 1.11 \\
\hline & T3 & 0.41 & 0.25 & 0.68 & 0.87 \\
\hline
\end{tabular}

def. - definition; GEE - generalized estimating equations; NDVI - Normalized Difference Vegetation Index; LCI - lower $95 \%$ confidence interval; OR - odds ratio; $\mathrm{T} 1-1^{\text {st }}$ tertile, $\mathrm{T} 2-2^{\text {nd }}$ tertile, $\mathrm{T} 3-3^{\text {rd }}$ tertile (see Table 3); UCI - upper confidence interval.

Boldface identifies significant associations $(\mathrm{p}<0.05)$.

All models were adjusted for age, sex, season of birth, parental atopy and parental education. 


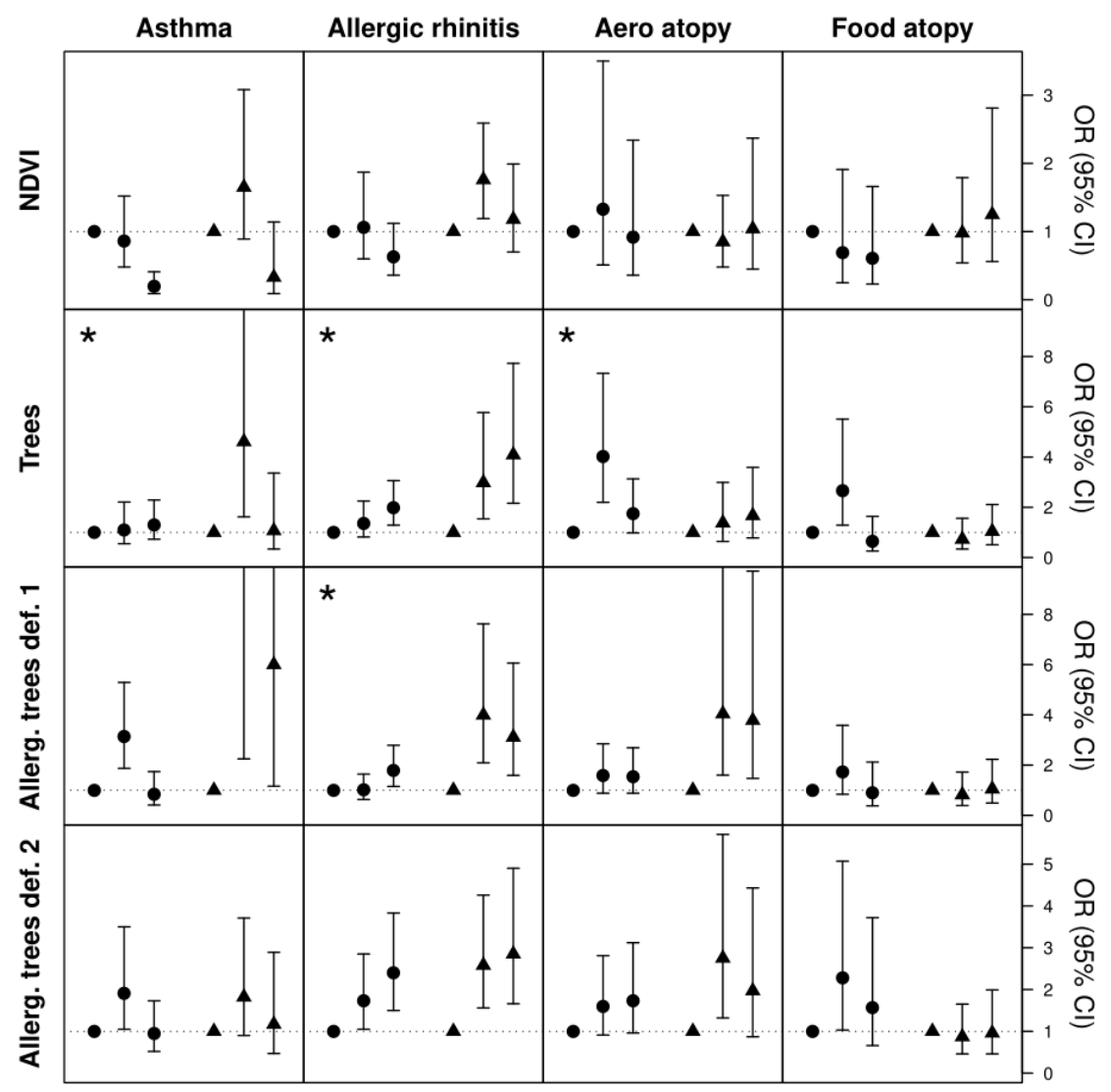

Figure 1. Associations between greenness, trees and allergenic trees in $500 \mathrm{~m}$ buffers around birth residential addresses with allergic outcomes up to 15 years, estimated by GEE models and stratified by $\mathrm{NO}_{2}$ levels. Circles represent the low tertile of $\mathrm{NO}_{2}$ while triangles represent the high tertile. An asterisk identifies a significant interaction term between the exposure variable and the continuous $\mathrm{NO}_{2}$ variables.

All models were adjusted for age, sex, season of birth, parental atopy and parental education.

Allerg. - allergenic; CI - confidence interval; def. - definition; OR - odds ratio; NDVI - Normalized Difference Vegetation Index; $\mathrm{NO}_{2}$ - nitrogen dioxide. 